\title{
Características emocionales de los adolescentes que han iniciado una vida sexual activa
}

\author{
Rangel-Hernández Monserrat, Martínez-Barrón Yanelly*, Perea-Ortíz María Guadalupe**, \\ Gallegos-Torres Ruth Magdalena***, Xeque-Morales Ángel Salvador**, \\ García-Aldeco Alejandra**** Palomé-Vega Gabriela**.
}

\section{Resumen}

- tos físicos como fisiológicos, caracterizada por inestabilidad

- emocional. Uno de los desafíos que deben de enfrentar los

- adolescentes es ejercer su sexualidad en todos sus ámbi-

- tos: psicológico, afectivo, es decir, en su desenvolvimiento

- como hombres o mujeres. Objetivo: identificar las emocio-

- nes de los adolescentes que han iniciado una vida sexual

- activa. Métodos: estudio descriptivo y transversal, realiza-

- do en una muestra de 155 adolescentes inscritos en una

- secundaria de la ciudad de Querétaro. Se excluyeron del

- estudio a aquellos estudiantes que no contaron con carta

- de consentimiento informado firmado por sus padres; se

- eliminaron también los instrumentos incompletos. Instru-

- mento elaborado ex profeso, validado y sometido a prueba

- piloto. Los datos fueron analizados mediante estadística

- descriptiva. Resultados: 61\% alumnos del sexo femenino;

- rango de edad de 12 a 15 años. 40\% de los participantes

- refirieron tener una vida sexual activa. $68 \%$ no planificó el

- primer encuentro sexual. El $8 \%$ se sintió presionado para

- iniciar relaciones sexuales. 61\% indicó que "el amor" no

- influyó en la decisión de iniciar una vida sexual y $51.6 \%$

- menciona que el "sexo" no es para unir a la pareja. Con-

- clusión: el estudio confirma el hecho de que la información

- sobre sexualidad que reciben los adolescentes es recibida

- mayormente por los amigos y que es necesario reforzar

- el aspecto educativo de los adolescentes con respecto a

- este fenómeno. LUXMÉDICA AÑO 10, NÚMERO 30, MAYO-AGOSTO

2015, PP 23-30

\section{Abstract}

Adolescence is a period of multiple changes, physical and physiological, characterized by emotional instability. One of the challenges of the adolescents is to practice their sexuality in all areas: psychological, affective, in their development as men or women. Objective: to identify the emotions of teenagers who started an active sexual life. Methods: descriptive and cross-sectional study, on a sample of 155 adolescents enrolled in a high school of Querétaro city. Were excluded from the study those students who did not have a letter of consent signed by their parents, incomplete instruments were also eliminated. An instrument prepared specially for the study was used and validated and pilot study. Data were analyzed using descriptive statistics. Results: $61 \%$ female students; age range of 12 to 15 years. $40 \%$ of the participants concerned have an active sexual life. 68\% not planned the first sexual encounter, $8 \%$ felt pressed to initiate active sexual life. $61 \%$ indicated that "love" did not influence the decision to start active sexual life and $51.6 \%$ mentions that "sex" does not to unite the couple. Conclusion: The study confirms the fact that the information about sexuality that adolescents receive is mainly by friends and that it is necessary to reinforce the educational aspect of adolescents with regard to this phenomenon. LUXMÉDICA AÑO 10, NÚMERO 30, MAYO-AGOSTO 2015, PP 23-30

* Pasante en Servicio Social, Licenciatura en Enfermería, Facultad de Enfermería, Universidad Autónoma de Querétaro. Querétaro, México. ** Profesores investigadores, Maestros en Ciencias de Enfermería. Facultad de Enfermería, Universidad Autónoma de Querétaro. Querétaro, México.

*** Profesora investigadora, Doctora en Ciencias de la Salud, Facultad de Enfermería, Universidad Autónoma de Querétaro. Querétaro, México.

**** Profesora investigadora, Maestra en Desarrollo Educativo, Facultad de Enfermería, Universidad Autónoma de Querétaro. Querétaro, México.

Fecha de recibido: 10 de abril de 2015

Fecha de aceptación: 7 de julio de 2015

Correspondencia: Dra. en C Ruth Magdalena Gallegos Torres, Facultad de Enfermería, Universidad Autónoma de Querétaro. Cerro de Las Campanas S/N, Las Campanas, 76010 Santiago de Querétaro, Querétaro, México. Teléfono: 01442192 1200. Correo electrónico: isisrmgx@gmail.com 


\section{Introducción}

La adolescencia está reconocida por diversos autores como la etapa más vulnerable y difícil de la vida de todo ser humano. En esta etapa ocurren complejos cambios, de índole biológica, psicológica y social, lo cual favorece el hecho de que el joven busque nuevas experiencias y aspiraciones ${ }^{1}$. Durante la adolescencia se alcanza la etapa final del crecimiento, con el comienzo de la capacidad de reproducción $y$, junto con ello, se inicia la inserción en el grupo de los adultos buscando, previo a esto, vivir nuevas experiencias, siendo una de ellas las vinculadas a la sexualidad. En este sentido, se reconoce que la identidad del adolescente está en proceso de maduración, así como también su desarrollo sexual, que incide en la necesidad de tener una separación de los padres. ${ }^{2}$

El inicio de la vida sexual se da junto con cambios físicos y psicológicos que permitirán alcanzar la capacidad de intimar sexualmente. Por un lado, los jóvenes completan el crecimiento y desarrollo, alcanzando la capacidad de reproducción y adquieren un pensamiento abstracto. Por otro, desarrollan autonomía emocional, una nueva identidad y modifican sus relaciones sociales, además de transformar la escala de valores por la que se evalúan a sí mismos y a los demás ${ }^{3}$. El desarrollo del adolescente, en general, se caracteriza por ser una etapa de fuerte transición y continua adaptación. El adolescente tendrá que definir, entre otras cosas, desde su nueva imagen corporal hasta su rol en un grupo. En múltiples ocasiones estas transformaciones generan rebeldía, necesidad de explorar nuevas sensaciones y dificultad para enfrentar frustraciones y angustias, por lo que quizás, por curiosidad o por broma, los adolescentes con facilidad pueden incurrir en diferentes errores' ${ }^{1}$.

De acuerdo con la Organización Mundial de la Salud (OMS), el ámbito de salud sexual y salud reproductiva es un área problemática a nivel mundial en los adolescentes. Entre los diversos problemas a los que se pueden enfrentar los adolescentes, sobre todo si carecen de la información sexual adecuada, están la falta de preparación y orientación para una conducta sexual responsable, la incapacidad para valorar el riesgo que implica una relación sexua ${ }^{14}$, los embarazos a temprana edad y las enfermedades de origen sexual. ${ }^{5}$

Otro factor que influye en esta problemática es el inicio temprano de relaciones sexuales, inicio que se encuentra entre los 13 y 15 años. Lo anterior se evidencia en los resultados de una investigación realizada en la ciudad de Bucaramanga, Colombia, en una población de 122 jóvenes, donde el 75\% de los estudiantes afirmaron haber sostenido relaciones sexuales antes de los 16 años ${ }^{6}$. En México, el inicio de relaciones sexuales a una temprana edad también ha sido confirmado. Se ha encontrado que el $16.1 \%$ de las 
adolescentes inicia su vida sexual entre los 12 y 19 años, siendo la edad promedio de inicio los 16.4 años ${ }^{5}$.

El inicio temprano de relaciones sexuales es una problemática sanitaria porque la primera relación sexual se correlaciona con una compleja red de características personales e influencias sociales que moldean dicha decisión ${ }^{5}$. El papel de la familia sin duda es importante en la adquisición de habilidades y por ende en el rol de protección o de riesgo ${ }^{7}$. Al referirse a los medios de obtener conocimientos acerca de la sexualidad, se indaga que la mayor parte de información se obtiene mediante las amistades, siendo el médico el que ocupa el último lugar?.

La interacción con sus padres, el abuso del alcohol y la presión de la pareja, en el caso de las niñas, influyen en la actividad sexual prematura y no deseada ${ }^{8}$. La evidencia científica muestra que el inicio de la actividad sexual a más temprana edad puede tener consecuencias psicológicas, sociales y económicas negativas; así mismo está asociado a un mayor número de parejas sexuales a lo largo de la vida ${ }^{8}$.

Además de la influencia cultural, las características de personalidad de un individuo influyen en la edad en que éste comienza a ejercer su sexualidad. La percepción que el adolescente tiene sobre sí mismo es, por lo general, muy coherente con su comportamiento externo y observable. Así, se ha relacionado en adolescentes la agresividad con sentimientos de percibirse en soledad e insatisfechos con su vida y la insatisfacción personal con la ansiedad e inestabilidad emocional ${ }^{9}$.

Con base en esto es que surge la necesidad de realizar esta investigación: el objetivo de ésta es comprender mejor el fenómeno del inicio de la vida sexual en adolescentes, indagar más acerca de los diversos componentes que intervienen en los conocimientos y las prácticas relacionadas con la sexualidad de los jóvenes, por lo que este estudio buscó identificar, de primera instancia, características emocionales de los adolescentes que han iniciado una vida sexual activa, de una escuela secundaria de la ciudad de Querétaro, México.

| | | | | | | | | | | | | | | | | | | | | | | | | | | | | | | | | | | | | | | | | | | | | | | | | | | | | | | | | | | | | | | | | | | | | | | | | | | | | | | | | | | | | | | | | | | | | | | | | | | | | | | |

\section{Material y métodos}

La investigación que se presenta fue un estudio descriptivo y transversal, realizado en adolescentes de una secundaria de la ciudad de Querétaro. El universo estuvo conformado por aproximadamente 1728 estudiantes, distribuidos en el turno matutino y vespertino. Para determinar el tamaño de la muestra, se utilizó la fórmula para poblaciones finitas, de manera tal que se encontró que la muestra debía estar conformada por 231 alumnos. 
El tipo de muestreo utilizado fue aleatorio, simple y estratificado. Se seleccionó a aquellos alumnos inscritos que cursaran el primer, segundo y tercer año, de ambos sexos y de ambos turnos. Una vez integrado el proyecto de investigación, éste fue evaluado y aprobado por el Comité de Investigación del Pregrado de la Licenciatura de Enfermería de la Facultad de Enfermería de la Universidad Autónoma de Querétaro. Dado que el instrumento de recolección de datos fue elaborado ex profeso para este estudio, fue sometido a validación por expertos, cinco personas, profesionales de la salud, investigadores y con grado de maestría. Una vez que se hicieron los ajustes requeridos al instrumento, éste se sometió a prueba piloto. El instrumento se denominó
"Test de sexualidad en adolescentes", se conforma de 55 preguntas, integradas por 19 ítems para datos socio demográficos y 36 para aspectos vinculados a aspectos emocionales y al ejercicio de la sexualidad. A excepción de los datos sociodemográficos, los ítems contaban con una escala Likert de 5 opciones de respuesta que van desde nunca hasta siempre. Para poder llevar a cabo el levantamiento de los datos, se solicitaron los permisos correspondientes con las autoridades de la escuela secundaria donde se trabajó. Vale la pena señalar que esta investigación se rigió bajo los principios éticos de la Investigación en seres humanos que establece la Ley General de Salud.

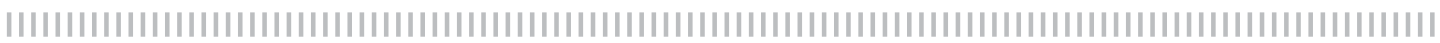

\section{Resultados}

A continuación se muestran los resultados de 155 alumnos. Para su mayor comprensión, los datos se mostrarán por categorías:

\section{Datos sociodemográficos}

Se identifica un rango de edad de los 12 a los 15 años, predominando aquellos de 15 (45\%). El 61\% (95) eran alumnos del sexo femenino. Con respecto a la religión, el $87 \%$ refieren practicar la religión católica; el 70\% tienen entre 1 y 3 hermanos, el $83 \%$ niega trabajar actualmente, por lo que sólo se dedican a estudiar.

\section{Familia-padres}

El 65\% (101) de los alumnos viven actualmente con ambos padres, por lo consiguiente, el estado civil de estos es de casados, con el 70\% (108). Dentro del nivel de estudios de los padres, se encontró que en el 46\% (72) de los casos, el padre sólo terminó la secundaria y que en el $47 \%$ (73) la madre terminó la secundaria. El 48\% (74) de alumnos respondió que ambos padres trabajan.

\section{Situación de pareja}

Sobre la situación de pareja, el 64\%(99) de los alumnos actualmente no tiene y de los que sí tienen, el 59\% (33) llevan un periodo de 6 meses o menos con la relación.

\section{Expresión de sentimientos}

Con respecto al sentir general de los estudiantes, el $48 \%$ de los alumnos respondieron que a veces han abandonado muchos de sus intereses y actividades; así mismo el $30 \%$ (46) rara vez expresa o muestra tristeza, soledad y desdicha; el 46.5\% (72) de los alumnos se encuentran irritables, de mal humor, negativos y difíciles de comprender; se sienten negativos consigo mismos el 41\% (64). 37\% (57) refirió tener poco respeto ante las figuras de autoridad. 


\section{Autopercepción}

En relación a cómo se perciben, al 54\% (84) les gusta su cuerpo. La mayor parte del tiempo se sienten deprimidos el $13 \%$ (20). Al 41\% (63) les gusta la interacción social, 34\% (53) no tienen miedo de estar solos, aunque $24 \%$ frecuentemente tiene este temor. $60 \%$ (93) de los alumnos consideran que la adolescencia no es la mejor etapa para tener relaciones sexuales.

\section{Socialización de la sexualidad}

Nunca hablan de sexo con su familia el $35 \%$ (54) y el $32 \%$ (50) ni con sus amigos. $19 \%$ (29) indicó no recibir información sobre sexualidad. 15\% (24) considera que constantemente piensa en sexo. $29 \%$ (45) de los estudiantes señaló que las personas que los rodean hablan de las relaciones sexuales que tienen con sus parejas, aunque $39 \%$ (60) señaló que sí se presenta esta situación al encontrarse con los amigos.
Factores para el inicio de una vida sexual $40 \%$ de los participantes, es decir, 62 alumnos, refirió que ya tiene una vida sexual activa. De éstos, el $8 \%$ se sintió presionado para tener relaciones sexuales con su pareja, aunque $14.5 \%$ indicó que sintió que su pareja estaba presionada para tener relaciones sexuales. $68 \%$ de los jóvenes tuvo su primera relación sexual sin haberla planificado, donde llama la atención que $61 \%$ indicó que no fue el amor el que influyó en esta decisión; así mismo, el $51.6 \%$ piensa que las relaciones sexuales no son para unir a la pareja. $58 \%$ de los participantes tuvieron la iniciativa de tener relaciones sexuales con su pareja. $27 \%$ se arrepintieron de su primera relación sexual. Los datos más sobresalientes por pregunta se muestran en la tabla 1 , la cual muestra las respuestas de siempre y frecuentemente que marcaron los alumnos, asimismo, se señala el año de secundaria donde sobresalieron los datos.

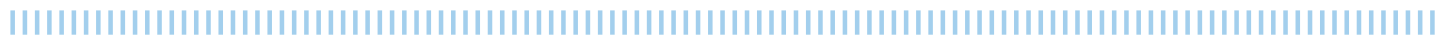

\section{Discusión}

Tras el análisis de los datos obtenidos en el estudio "Características emocionales de los adolescentes que han iniciado una vida sexual activa", se encontró que en mayor porcentaje las alumnas $(7.4 \%)$ hablan de sexo con su familia a diferencia de los hombres $(5 \%)$, lo cual coincide con un estudio realizado en la Ciudad de México en el 2011 por Burgos ${ }^{10}$, en adolescentes de preparatoria, donde se comparó una escuela rural con una urbana; se observó que el mayor porcentaje en alumnas (79\%), respecto de los alumnos $61 \%$ ) consideran haber recibido información sexual de sus papás; en el caso de la rural el porcentaje de las alumnas que afirmaron tener información sexual de sus papás sigue siendo mayor que el de los alumnos. Sin embargo, contrario a lo anterior, González et al. ${ }^{8}$ menciona que en su estudio, el mayor porcentaje de los jóvenes indicó que el conocimiento refieren haberlo adquirido a través de la televisión con un $56.4 \%$, asimismo se considera muy pobre el papel de la escuela, la familia y el personal de salud en la preparación de los adolescentes en aspectos relacionados con la sexualidad. 


\section{Tabla I}

\section{Resultados sobresalientes del instrumento y año de secundaria en que hubo mayor prevalencia}

\begin{tabular}{|c|c|c|}
\hline Pregunta & Frecuentemente & Siempre \\
\hline & $\mathbf{M \%}$ & $\mathbf{M \%}$ \\
\hline Expreso o muestro tristeza, soledad, desdicha la mayor parte del tiempo & $\begin{array}{l}1.7 \% \quad 13.7 \% \\
\text { 3er año }\end{array}$ & $\begin{array}{l}0.5 \% 12.6 \% \\
1 \text { er año }\end{array}$ \\
\hline $\begin{array}{l}\text { Frecuentemente me encuentro irritable, de malhumor, negativo y soy difícil } \\
\text { de comprender }\end{array}$ & $\begin{array}{l}10.6 \% 11.6 \% \\
2 \text { do año } 1 \text { er año }\end{array}$ & $12.6 \%$ \\
\hline Frecuentemente me siento negativo conmigo mismo & $\begin{array}{c}5.0 \% \quad 6.3 \% \\
\text { 3er año }\end{array}$ & $\begin{array}{l}6.7 \% \quad 10.5 \% \\
1 \text { er año }\end{array}$ \\
\hline Tengo dificultad para dormir & $\begin{array}{c}5.0 \% \text { 7.4\% } \\
2 \text { do año }\end{array}$ & $\begin{array}{c}5.0 \% \text { 7.4\% } \\
1 \text { er año }\end{array}$ \\
\hline Tengo mucho miedo de estar solo & $\begin{array}{c}13.3 \% \quad 13.7 \% \\
1 \text { er año }\end{array}$ & $\begin{array}{l}11.7 \% \quad 9.5 \% \\
2 \text { do año }\end{array}$ \\
\hline Estoy muy deprimido & $\begin{array}{l}1.7 \% \quad 13.7 \% \\
\text { 3er año }\end{array}$ & $\begin{array}{c}3.3 \% \text { 4.2\% } \\
1 \text { er año }\end{array}$ \\
\hline Tengo que estar siempre en una relación sentimental & $1.7 \% \quad 3.2 \%$ & $1.7 \%$ \\
\hline Creo que la adolescencia es la mejor etapa para tener relaciones sexuales & $\begin{array}{l}10 \% \quad 2.1 \% \\
\text { 3er año }\end{array}$ & $\begin{array}{l}3.3 \% \text { B. } 3 \% \\
\text { 3er año }\end{array}$ \\
\hline Hablo de sexo con mi familia & $\begin{array}{l}6.7 \% \text { 6.3\% } \\
1 \mathrm{er} \text { año }\end{array}$ & $\begin{array}{l}5.0 \% \text { i } 7.4 \% \\
\text { 3er año }\end{array}$ \\
\hline Hablo de sexo con mis amigos & $\begin{array}{c}13.3 \% \text { 8.4\% } \\
\text { 3er año }\end{array}$ & $\begin{array}{c}13.3 \% \quad 13.7 \% \\
\text { 3er año }\end{array}$ \\
\hline Considero que tener una sola pareja es lo conveniente & $\begin{array}{c}18.3 \% 12.6 \% \\
\text { 3er año }\end{array}$ & $\begin{array}{l}51.7 \% \quad 49.5 \% \\
2 \text { do año }\end{array}$ \\
\hline Pienso constantemente en el sexo & $\begin{array}{l}5.0 \% \text { 3.2\% } \\
\text { 3er año }\end{array}$ & $\begin{array}{l}5.0 \% \text { 1.1\% } \\
\text { 3er año }\end{array}$ \\
\hline $\begin{array}{l}\text { Considero que es necesario tener relaciones sexuales para ser aceptado } \\
\text { por mis amigos }\end{array}$ & $2.1 \%$ & $1.7 \%$ \\
\hline Anhelo tener relaciones íntimas (pero no las suelo tener) & $\begin{array}{c}1.7 \% \text { 2.1\% } \\
2 \text { do año }\end{array}$ & $\begin{array}{l}3.3 \% \text { 1.1\% } \\
\text { 3er año }\end{array}$ \\
\hline Me sentí presionada(o) por mi pareja para tener relaciones sexuales & $\begin{array}{l}0 \% \text { 2.6\% } \\
1 \text { er año }\end{array}$ & $\begin{array}{l}02.6 \% \\
2 \text { do año }\end{array}$ \\
\hline Pienso que mi pareja se sintió presionada para tener relaciones sexuales & $\begin{array}{c}8.7 \% \quad 2.6 \% \\
1 \text { er año } \\
\end{array}$ & $\begin{array}{l}4.3 \% \text { or } 0 \% \\
1 \text { er año }\end{array}$ \\
\hline Planifiqué mi primera relación sexual & $\begin{array}{c}0 \% \quad 2.6 \% \\
1 \text { er año }\end{array}$ & $\begin{array}{l}8.7 \% \text { \% } 5.1 \% \\
\text { 3er año }\end{array}$ \\
\hline El amor que tengo por mi pareja influyo para tener relaciones sexuales & $\begin{array}{c}13 \% \quad 7.7 \% \\
\text { 3er año }\end{array}$ & $\begin{array}{l}13 \% \text { i. } 7.7 \% \\
\text { 3er año }\end{array}$ \\
\hline Tomé la iniciativa para tener relaciones sexuales con mi pareja & $\begin{array}{c}4.3 \% \quad 0 \% \\
1 \text { er año }\end{array}$ & $\begin{array}{c}13 \% \quad 10.3 \% \\
\text { 3er año }\end{array}$ \\
\hline Expreso mis sentimientos con mi pareja & $\begin{array}{l}8.7 \% \text { 10.3\% } \\
\text { 3er año }\end{array}$ & $\begin{array}{l}43.5 \% \text { 33.3\% } \\
2 \text { do año }\end{array}$ \\
\hline Tomo las relaciones sexuales como algo que une a las parejas & $\begin{array}{c}21.7 \% \quad 7.7 \% \\
1 \text { er año }\end{array}$ & $\begin{array}{l}13 \% \text { a } 5.1 \% \\
\text { 3er año }\end{array}$ \\
\hline Cuando tuve mi primera relación sexual me sentí arrepentido & $\begin{array}{l}4.3 \% \quad 2.6 \% \\
2 \text { do año }\end{array}$ & $\begin{array}{c}4.3 \% \text { 5.1\% } \\
\text { 3er año }\end{array}$ \\
\hline
\end{tabular}

Fuente: instrumento aplicado, 2014

Al respecto, Naranjo et al. ${ }^{7}$ mencionan que "fue importante conocer cómo aún el medio social extra familiar donde se desenvuelve el adolescente sigue siendo el principal aporte de información en el tema de la sexualidad, principalmente los amigos, $94.0 \%$ respectivamente relegando a la familia $(10.4 \%)$ y la labor del médico de la familia (7.4\%) a último lugar". Así los datos de este estudio y el de Burgos ${ }^{10}$, 
muestran que los adolescentes sí obtienen la información sobre la sexualidad con la familia, mientras que otros autores como González ${ }^{8}$ y Naranjo no encontraron este dato?.

Sobre la vida sexual, en esta investigación se encontró que el $40 \%$ de estudiantes ya la ha iniciado, datos que son similares a los encontrados por Parra ${ }^{11}$, en su estudio realizado con 277 adolescentes, de los cuales $37 \%$ ya tiene vida sexual activa, aunque en el caso de Ruiz-Canela ${ }^{12}$, realizado con 2615 estudiantes, el porcentaje es menor, siendo de $24.4 \%$.

En relación en si se sintieron presionados para tener relaciones sexuales, los adolescentes contestaron $2.6 \%$ que siempre; al preguntarles si piensan que su pareja se sintió presionada el $8.7 \%$ mencionan que frecuentemente. En este sentido, Díaz et $\mathrm{al}^{13}$ señalan que la presión entre los pares para tener relaciones sexuales es percibida por los adolescentes como nada en un $66 \%$, un poco fue mencionado por el $21 \%, 6 \%$ considera que existe una buena cantidad de presión y por último existe un $2 \%$ que reporta mucha presión para iniciar la vida sexual.

Con respecto a lo emocional se planteó la siguiente pregunta: ¿Frecuentemente me encuentro irritable, de malhumor, negativo y soy difícil de comprender? El $12.6 \%$ de los adolescentes contestaron que siempre, este resultado tiene similitud con lo que dice Orcasita et al. ${ }^{6}$ donde menciona que bajo el nivel de significación del $5 \%$, con la prueba no paramétrica de Mann-Whitney, se encontraron diferencias significativas entre los adolescentes que han iniciado sus relaciones sexuales y los que no han iniciado en cuanto al apoyo emocional ( $p$-valor $=0.02)$ e instrumental ( $p$-valor $=0.001$ ), es decir, que los adolescentes que iniciaron su vida sexual perciben un apoyo emocional e instrumental, mucho más bajo que los que no han iniciado su vida sexual; así se puede observar que los adolescentes que ya iniciaron su vida sexual sí tienen un problema emocional que repercute en su toma de decisiones.

Ramírez y Almeida ${ }^{14}$ mencionan que en la variable percepción hacia las relaciones sexuales se obtuvo un puntaje promedio de 5.1; es posible deducir que hay $73.3 \%$ de respuestas en que se acepta la práctica sexual como un acto natural y permisible, esto tiene relación con lo que encontramos sobre si los adolescentes piensan que tener relaciones sexuales es algo que une a la pareja, donde el $21.7 \%$ responde que frecuentemente y el $13 \%$ que siempre, ya que la percepción de las relaciones sexuales son lo más natural entre un hombre y una mujer y sirve para unir a las parejas.

\section{Conclusiones}

La primera aportación del estudio frente a los resultados es señalar una vez más que el conocimiento que tienen los adolescentes sobre la sexualidad no se obtiene en la familia si no bien en el entorno de los amigos.

Al procurar identificar las características del inicio de las relaciones sexuales y las emociones que han presentado los adolescentes, se encontró que son escasas las muestras de tristeza y soledad, aunque más del $40 \%$ manifestaron sentirse irritables, de mal humor, negativos y difíciles de comprender. Se reconoce que necesita hacerse un sondeo más profundo para valorar el significado que da el adolescente al inicio de su vida sexual. 
Sin embargo, se identificó que aparentemente los adolescentes sólo toman las relaciones sexuales como un pasatiempo o un juego. En el estudio se apreció que hoy en día los adolescentes no tienen la suficiente madurez para tener una relación de pareja y pocos saben cómo es que deben de cuidarse cuando ya iniciaron una vida sexual activa, esto de acuerdo a los resultados reflejados en múltiples investigaciones que se encuentran en la literatura.

\section{Bibliografía}

1. Pérez Pérez $M$, Sosa Zamora $M$, Amarán Valverde JE, del Rosario Pinto M, Arias Acosta D. Algunos aspectos relacionados con el conocimiento sobre sexualidad en adolescentes de un área de salud. Medisan. 2009;13(3):0-0.

2. Loayssa JR, Echagüe I. La salud en la adolescencia y las tareas de los servicios de salud. Anales del Sistema Sanitario de Navarra [Internet]. 2009 [citado 29 de enero de 2014]. p. 93-105. Recuperado a partir de: http://arsenopirita.boumort.cesca.cat/index.php/ ASSN/article/view/5902.

3. Belandia $M$, Durán $P$, Penzo $M$, Martínez $G$, Bazano $M$, Maddaleno $M$, et al. Sistema informático del adolescente (SIA): historia clínica del adolescente y formularios complementarios: instrucciones de llenado y definición de términos; Adolescent information system (SIA): adolescent clinical record and complementary forms: filling instructions and definition of terms. CLAPSMR Publ Científica [Internet]. 2010 [citado 28 de enero de 2014];(1579). Recuperado a partir de: http://bases.bireme.br/cgi-bin/wxislind. exe/iah/online/?IsisScript $=$ iah/iah. $x i s \& s r c=$ google \&base $=$ ADOLEC\&lang $=p \&$ nextAction $=\operatorname{lnk} \&$ exprSe $\operatorname{arch}=586902 \&$ indexSearch $=$ ID .

4. De la Merced Ayala-Castellanos M, VizmanosLamotte B, Portillo-Dávalos RA. Salud sexual y reproductiva en adolescentes de escuela secundaria en Guadalajara, México. Ginecol Obstet Mex. 2011;79(2):86-92.

5. Esquivel Alcocer LA, Casanova Quiñones GF, Uc Aké NR. Conocimientos de la sexualidad en estudiantes de preparatoria. Rev Educ Cienc [Internet]. 2011 [citado 13 de marzo de 2013];5(23). Recuperado a partir de: http://educacionyciencia.org/index.php/ educacionyciencia/article/view/160.

6. Orcasita LT, Uribe AF, Castellanos LP, Rodríguez MG. Apoyo social y conductas sexuales de riesgo en adolescentes del municipio de Lebrija-Santander. Rev Psicol. 2012;30(2):371-406.

7. Naranjo RA, Reyes AO, García RG, León JM, Naranjo LM. Adolescencia e inicio precoz de las relaciones sexuales. Algunos factores desencadenantes. Gac Med Espirituana. 2008;10(2):1-5.

8. González E, Montero A, Martínez V, Mena P, Varas $M$. Percepciones y experiencias del inicio sexual desde una perspectiva de género, en adolescentes consultantes en un centro universitario de salud sexual y reproductiva. Rev Chil Obstet Ginecol. 2010;75(2):84-90.

9. Silva IL. Adolescentes y percepción del sí mismo: la construcción de una imagen realista de la adolescencia desde la familia y la escuela. Etic Net. 2011;9(11):233-52.

10. Burgos Fajardo RJ. Sexualidad y adolescencia: hacia una educación familiar integral. Rev Educ Cienc [Internet]. 2011 [citado 4 de junio de 2013];1(16). Recuperado a partir de: http://educacionyciencia.org/ index.php/educacionyciencia/article/viewFile/117/ pdf.

11. Parra Villarroel J, Domínguez Placencia J, Maturana Rosales J, Pérez Villegas R, Carrasco Portiño M Conocimiento y percepción de adolescentes sobre el servicio de planificación familiar en Chile. Salud Colect. 2013;9(3):391-400.

12. Ruiz-Canela M, López-del Burgo $C$, Carlos S, Calatrava $M$, Osorio $A$, de Irala J. Familia, amigos y otras fuentes de información asociadas al inicio de las relaciones sexuales en adolescentes de El Salvador. Rev Panam Salud Publica. 2012;31(1):54-61.

13. Díaz Rodríguez CL, González Ramírez MT. Conductas problema en adolescentes en la ciudad de Monterrey, México. Enferm Glob. 2014;13(33):1-16.

14. Rivera CAR, Félix GIA. Percepción hacia las relaciones sexuales y riesgo de embarazo en adolescentes. Psicol Salud. 2014;13(2):227-33. 